Research Article

\title{
Rough Set Neural Network Feature Extraction and Pattern Recognition of Shaft Orbits Based on the Zernike Moment
}

\author{
Xinfeng Ge $\mathbb{D}^{1},{ }^{1}$ Jing Zhang, ${ }^{1}$ Ye Zhou $\mathbb{D}^{2},{ }^{2}$ Jianguo Cai, ${ }^{3}$ Hui Zhang, ${ }^{3}$ Hongchang Hua, \\ Dong Chen, ${ }^{1}$ Ming Zhao, ${ }^{4}$ Jinqi Du, ${ }^{5}$ and Yuan Zheng ${ }^{1}$ \\ ${ }^{1}$ College of Energy and Electric Engineering, Hohai University, Nanjing 211100, China \\ ${ }^{2}$ Institute for Hydraulic Machinery, China Institute of Water Resources and Hydropower Research, Beijing 100038, China \\ ${ }^{3}$ Chongqing Shipping Construction Development Co., Ltd., Chongqing 500000, China \\ ${ }^{4}$ Research Institute of Yunnan Power Grid Co. Ltd., Kunming, China \\ ${ }^{5}$ Yunnan Electric Test \& Research Institute Group. Co. Ltd, Kunming, China \\ Correspondence should be addressed to Xinfeng Ge; gexinfeng@hhu.edu.cn
}

Received 28 December 2020; Revised 25 March 2021; Accepted 21 April 2021; Published 29 April 2021

Academic Editor: Ling Zhou

Copyright ( $\odot 2021$ Xinfeng Ge et al. This is an open access article distributed under the Creative Commons Attribution License, which permits unrestricted use, distribution, and reproduction in any medium, provided the original work is properly cited.

\begin{abstract}
In the shaft axis monitoring of hydrogenerating unit condition monitoring and fault diagnosis, the shaft orbit is intuitive and comprehensively reflects the unit operation state, and different shaft orbits correspond to different fault types, which can accurately indicate a system vibration fault. Shaft orbit identification has important significance for vibration fault diagnosis. In getting the feature extraction and pattern recognition of a shaft orbit, the Zernike moment is better than the Hu moment; it has the advantages of a small calculation error and a high recognition rate. A rough set neural network (RS-BP hybrid model) of shaft orbit recognition is established, which uses just 13 moment eigenvalues reserved by the rough set feature selection algorithm as input variables; it has the same calculation error and recognition rate and reduces the calculation time step. The simulation of the recognition of shaft orbits shows that the hybrid model has achieved good results in the identification of shaft orbits.
\end{abstract}

\section{Introduction}

Because of the complex operating conditions involved, there are many factors that cause the instability of hydropower units, including hydraulic, mechanical, and electromagnetic factors. Among these three important factors, hydraulic vibration is the excitation source that has a crucial influence on the vibration of the unit. If the low-frequency oscillation is induced by the vibration of the power system, it will affect the stable operation of the power grid $[1,2]$. Through hydraulic excitation, each component of the unit exhibits different vibration characteristics. During the shafting monitoring, the shaft orbit directly and comprehensively reflects the operation state and vibration fault of the unit, and different shaft orbits usually reflect different faults. The hydroelectric unit condition monitoring system can obtain many operating characteristics of the unit from the axis orbit of the unit, so the recognition of the shaft orbit plays an important role in the fault diagnosis.

In recent years, many experts have performed theoretical research and simulation analysis on hydrogenerator shaft orbits, using different methods to research the recognition of shaft orbits [3-7]. At present, the recognition of shaft orbits mainly includes precession direction recognition and shape recognition. Shape recognition of shaft orbits mainly includes feature extraction and pattern recognition. The feature extraction methods include Fourier descriptors [8-11], Hu moments [12-15], and Zernike moments [16], as well as classification of the data with an advanced pattern recognition method [17]. Kauppinen et al. [18] tried to use autoregressive modeling and Fourier descriptors of closed contours to recognize the typical shape of shaft orbits. This showed better performance for Fourier-based methods, especially for images containing noise. Fu et al. [8] combined a BP neural network and modified Fourier descriptors to 
classify the shaft orbits for fault diagnosis of the turbine rotor. The simulation results were for the automatic identification of three typical shaft orbits. In order to obtain the characteristics of the axis orbit, the edge acquisition algorithm is typically used, and, by contrasting each edge extraction algorithm, the applicability of the corresponding algorithm is obtained. Then the algorithm is improved according to the corresponding boundary features. An intelligent model, such as the neural network model, is established to achieve the intelligent classification of faults in the mechanical system.

Fourier descriptors have the advantage of feature extraction for completely closed curves, but the shaft orbits' coincidence performance for the hydropower unit is so poor that it is hardly closed, so this paper uses Hu moments and Zernike moments to extract features of the shaft orbit.

For intelligent pattern recognition, this paper adopts the common BP neural network and applies the rough set theory to simplify the neural network with overly large input samples and a complex network structure.

\section{Formation and Characteristics of the Shaft Orbit}

In the hydrogenerating unit stability test and application of the monitoring system, two eddy current displacement sensors are orthogonally installed in the guide bearing (upper and lower guide) horizontal section. This ensures that the vibration signal of two mutually perpendicular directions can be measured. Then the high-frequency noise of the vibration signal is eliminated using a low pass filter, and finally a center orbital path is formed using the analogto-digital converter. As shown in Figure 1, two shaft swing sensors are installed in the unified plane of the rotating shaft. The position of the rotor center can be determined by the outputs $x(t)$ and $y(t)$ of the two sensors at the $t$ moment, and then the position of the rotor center at different time can be transformed into a curve, which is the vibration track of the rotor in the bearing, called the shaft orbit.

The shaft orbit of different shapes reflects the operation of different units, and its shape is an important basis for judging the running state and failure of the shaft, which implies the fault information of the unit. Table 1 shows the shaft orbit shapes and the potential failure types, and Figure 2 shows typical faults.

\section{Moment Feature Extraction and Model Establishment}

\subsection{Hu Moments and Improvement}

3.1.1. $\mathrm{Hu}$ Moments. $\mathrm{Hu}$ moment theory has been widely used in the field of image recognition. It is a method of extracting image features based on a statistical feature. Two-dimensional moments are often used in digital image recognition. In fact, it extends the order moments of onedimensional signals to two-dimensional space. By using normalization, we obtain the moment's characteristic quantities that are sensitive to the change of shape, while the translation, rotation, and scaling of two-dimensional images remain unchanged. $\mathrm{Hu}$ [19] constructed 7 moments with rotation, translation, and scale invariance in 1962. In the field of image recognition, a statistical feature extraction method was established using moment theory, and it was widely used. In 1978, Wong and Hall [20] proved that the invariance of the $\mathrm{Hu}$ moment could be guaranteed when the scaling factor $\rho \leq 2$ and the rotation angle of the trajectory graph $\theta \leq 45^{\circ}$. For a two-dimensional function $f(x, y) \in\left(R^{2}\right)$, which is defined on the plane $o-x y$, the $p+q$ order mixed origin moment is defined according to the following formula:

$$
p, q=0,1,2 \ldots
$$

The $p+q$ order mixed central moment is defined according to the following formula:

$$
\begin{aligned}
& \mu_{p q}=\int_{-\infty}^{+\infty} \int_{-\infty}^{+\infty}(x-\bar{x})^{p}(y-\bar{y})^{q} f(x, y) \mathrm{d} x \mathrm{~d} y, \\
& p, q=0,1,2 \ldots
\end{aligned}
$$

where $(\bar{x}, \bar{y})$ is the gray center of gravity for the image $f(x, y)$.

$$
\begin{aligned}
& \bar{x}=\frac{m_{10}}{m_{00}} \\
& \bar{y}=\frac{m_{01}}{m_{00}} .
\end{aligned}
$$

The normalized central moment is defined as

$$
\eta_{p q}=\frac{\mu_{p q}}{\mu_{00}^{r}} .
$$

Under the constraints, $r=(p+q+2) / 2 ; p+q=$ $0,1,2,3 \ldots$

Ming-kuei $\mathrm{Hu}$ proposed the construction method of moments in 1962. By using the above central moments, 7 moment functions are constructed. These seven expressions correspond to translation, scaling, and rotation.

$$
\begin{aligned}
\phi_{1}= & \eta_{20}+\eta_{02}, \\
\phi 2= & \left(\eta_{20}-\eta_{02}\right)^{2}+4 \eta_{11}^{2}, \\
\phi_{3}= & \left(\eta_{30}-3 \eta_{12}\right)^{2}+\left(\eta_{03}-3 \eta_{21}\right)^{2}, \\
\phi_{4}= & \left(\eta_{30}+\eta_{12}\right)^{2}+\left(\eta_{03}+\eta^{21}\right)^{2}, \\
\phi_{5}= & \left(\eta_{30}-3 \eta_{12}\right)\left(\eta_{30}+\eta_{12}\right)\left[\left(\eta_{30}+\eta_{12}\right)^{2}-3\left(\eta_{03}+\eta_{21}\right)^{2}\right. \\
& +\left(3 \eta_{21}-\eta_{03}\right)\left(\eta_{03}+\eta_{21}\right)\left[3\left(\eta_{30}+\eta_{12}\right)^{2}-\left(\eta_{03}+\eta_{21}\right)^{2}\right], \\
\phi_{6}= & \left(\eta_{20}-\eta_{02}\right)\left[\left(\eta_{30}+\eta_{12}\right)^{2}-\left(\eta_{03}+\eta_{21}\right)^{2}\right] \\
& +4 \eta_{11}\left(\eta_{30}+\eta_{12}\right)\left(\eta_{03}+\eta_{21}\right), \\
\phi_{7}= & \left(3 \eta_{21}-\eta_{03}\right)\left(\eta_{30}+\eta_{12}\right)\left[\left(\eta_{30}+\eta_{12}\right)^{2}-3\left(\eta_{03}+\eta_{21}\right)^{2}\right] \\
& +\left(3 \eta_{12}-\eta_{30}\right)\left(\eta_{03}+\eta_{21}\right)\left[3\left(\eta_{30}+\eta_{12}\right)^{2}-\left(\eta_{03}+\eta_{21}\right)^{2}\right] .
\end{aligned}
$$




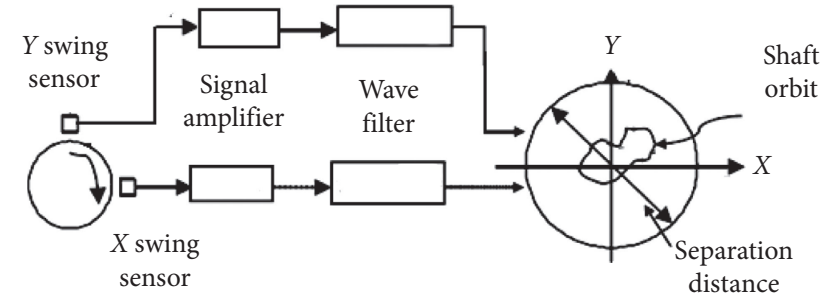

Figure 1: Measurement of the shaft orbit.

TABLE 1: Shaft orbit shapes and potential failure types.

\begin{tabular}{lcc}
\hline No. & Shaft orbits & Failure type \\
\hline 1 & Ellipse & Unbalanced or main axis is not \\
2 & straight \\
2 & Banana and outer " 8 " & Misalignment \\
3 & Petal shape & Vortex band of draft tube \\
4 & Inner " 8 " & Oil whirl \\
5 & Two separate ellipses & Bistability \\
6 & Protruding burr & Static and dynamic component \\
\hline
\end{tabular}

3.1.2. Moments in Discrete States. The $p+q$ order origin moment and central moment of a two-dimensional image function $f(x, y)$ in a discrete state are defined according to the following formulas:

$$
\begin{aligned}
m_{p+q} & =\sum_{i=1}^{N} \sum_{j=1}^{M} x_{i}^{p} y_{j}^{q} f\left(x_{i}, y_{i}\right), \\
\mu_{p+q} & =\sum_{i=1}^{N} \sum_{j=1}^{M}\left(x_{i}-\bar{x}\right)^{p}\left(y_{j}-\bar{y}\right)^{q} f\left(x_{i}, y_{i}\right) .
\end{aligned}
$$

Under the constraints,

$$
\begin{gathered}
\bar{x}=\frac{\sum_{i=1}^{N} \sum_{j=1}^{M} x_{i} f\left(x_{i}, y_{i}\right)}{\sum_{i=1}^{N} \sum_{j=1}^{M} f\left(x_{i}, y_{i}\right)}, \\
\bar{y}=\frac{\sum_{i=1}^{N} \sum_{i=1}^{M} y_{i} f\left(x_{i}, y_{i}\right)}{\sum_{i=1}^{N} \sum_{j=1}^{M} f\left(x_{i}, y_{i}\right)} .
\end{gathered}
$$

Normalized central moments in discrete states, which are defined by formulas (8) and (9), can clearly show that the moment functions in discrete states are also translationindependent. By observing the definition of the central moment, it is found that the central moment may be negative. Because the change range of moment $s$ is large, data compression can be achieved by the logarithmic processing of moments. Since the moments may appear negative, we need to take the absolute value before doing logarithmic operations. The actual moments can be obtained by means of a logarithm:

$$
M_{i}=\log _{10}\left|\varphi_{i}\right|, \quad i=1,2,3,4,5,6,7 .
$$

3.1.3. Improvement of Moments. If the scaling factor of the trajectory graph is $\rho$, the relationship between the moment $\phi_{i}^{\prime}$ in discrete states after scaling and the moment $\phi_{i}$ of the original graph is shown with the following formula:

$$
\begin{aligned}
\phi_{1}^{\prime} & =\rho^{2} \phi_{1}, \\
\phi_{2}^{\prime} & =\rho^{4} \phi_{2}, \\
\phi_{3}^{\prime} & =\rho^{6} \phi_{3}, \\
\phi_{4}^{\prime} & =\rho^{6} \phi_{4}, \\
\phi_{5}^{\prime} & =\rho^{12} \phi_{5}, \\
\phi_{6}^{\prime} & =\rho^{8} \phi_{6}, \\
\phi_{7}^{\prime} & =\rho^{12} \phi_{7} .
\end{aligned}
$$

In order to make these moment functions independent of scaling, we can consider the elimination of the scaling factor and use $\phi_{2}^{\prime}$ as the benchmark to find the final expression of the rest moment functions obtained by this moment function.

$$
\begin{aligned}
& M_{1}^{*}=\left(\frac{\phi_{1}^{\prime}}{\phi_{2}^{\prime}}\right)=\left(\frac{\left(\phi_{1}\right)^{2}}{\phi_{2}}\right), \\
& M_{3}^{*}=\left(\frac{\left(\phi_{3}^{\prime}\right)^{2}}{\left(\phi_{2}^{\prime}\right)^{3}}\right)=\left(\frac{\left(\phi_{3}\right)^{2}}{\left(\phi_{2}\right)^{3}}\right), \\
& M_{4}^{*}=\left(\frac{\left(\phi_{4}^{\prime}\right)^{2}}{\left(\phi_{2}^{\prime}\right)^{3}}\right)=\left(\frac{\left(\phi_{4}\right)^{2}}{\left(\phi_{2}\right)^{3}}\right), \\
& M_{5}^{*}=\frac{\phi_{5}^{\prime}}{\left(\phi_{2}^{\prime}\right)^{3}}=\frac{\phi_{5}}{\left(\phi_{2}\right)^{3}}, \\
& M_{6}^{*}=\frac{\phi_{6}^{\prime}}{\left(\phi_{2}^{\prime}\right)^{2}}=\frac{\phi_{6}}{\left(\phi_{2}\right)^{2}}, \\
& M_{7}^{*}=\frac{\phi_{7}^{\prime}}{\left(\phi_{2}^{\prime}\right)^{3}}=\frac{\phi_{7}}{\left(\phi_{2}\right)^{3}} .
\end{aligned}
$$

3.2. Zernike Moment. Zernike moment is one kind of special complex moments, which are based on orthogonal functions called Zernike polynomials. Compared with HU moment, the computation of Zernike moments is more complex, but they have greater advantages in terms of their expressive power and noise sensitivity. Zernike moments have the characteristics of rotation invariance and have been widely used in the field of target recognition.

3.2.1. Definition of Zernike Moments. Zernike [21] presents a set of orthogonal polynomials $V_{n m}(\rho, \theta)$, which are orthogonal in the unit circle $(\rho \leq 1)$. The polynomial function in polar coordinates is obtained as follows:

$$
V_{n m}(\rho, \theta)=R_{n m}(\rho) \exp (\operatorname{im} \theta) .
$$




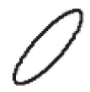

(a)

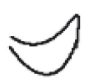

(b)

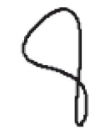

(c)

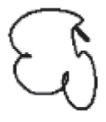

(d)

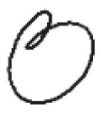

(e)

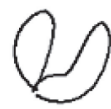

(f)

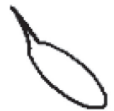

(g)

Figure 2: Shaft orbit of typical faults. (a) Ellipse. (b) Banana. (c) Outer "8." (d) Petal shape. (e) Inner "8." (f) Two separate ellipses. (g) Protruding burr.

Under the constraints, $n=0,1,2 \ldots$ and $m=0$, $\pm 1, \pm 2, \ldots .,|m| \leq n$, where $(n-|m|)$ is even number, $\rho$ is vector from origin point to pixel point $(x, y), \theta$ is the anticlockwise angle between the vector $\rho$ and the $x$-axis, and $R_{n m}(\rho)$ is real-valued radial polynomial.

$$
\begin{aligned}
R_{n m}(\rho) & =\sum_{s=0}^{(n-m \mid) / 2}(-1)^{s}, \\
& \frac{(n-s) ! \rho^{(n-2 s)}}{s !((n-2 s+|m|) / 2) !((n-2 s-|m|) / 2) !}
\end{aligned}
$$

Radial polynomials $R_{n m}(\rho)$ satisfy the following relationship:

$$
\int_{0}^{1} R_{n l}(r) R_{m l}(r) \rho \mathrm{d} \rho=\frac{1}{2(n+1)} \delta_{n m}
$$

where $\delta_{n m}$ is the Kronecker delta.

The orthogonality conditions of Zernike polynomials are as follows:

$$
\int_{0}^{2 \pi} \int_{0}^{1} V_{n l}^{*}(r) V_{m k}(\rho, \theta) \rho \mathrm{d} \rho \mathrm{d} \theta=\frac{1}{2(n+1)} \delta_{n m} \delta_{l k},
$$

where $*$ denotes the complex conjugate. The following formula can be obtained from formula (16):

$$
V_{n m}^{*}(\rho, \theta)=V_{n,-m}(\rho, \theta) .
$$

Some important properties of $R_{n m}(\rho)$ can be obtained from the following formula:

$$
\begin{aligned}
& R_{n m}(1)=1, R_{n n}(r)=\rho^{n}, \\
& R_{00}(\rho)=1 .
\end{aligned}
$$

An $n$ order Zernike polynomial with a repetition rate of $M$ is defined according to the following formula:

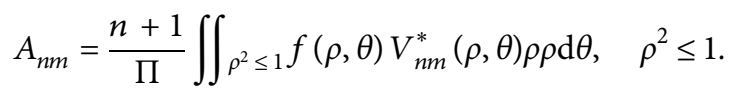

For a digital image, the integral is replaced by a summation, namely,

$$
A_{n m}=\frac{n+1}{\pi} \sum_{x} \sum_{y} f(x, y) V_{n m}^{*}(\rho, \theta) \mathrm{d} x \mathrm{~d} y, \quad x^{2}+y^{2} \leq 1 .
$$

To calculate the Zernike moments of an axis shaft orbit, it is necessary to move the center of the axis trajectory image to the coordinate circle and map the pixel points of the trajectory into the unit circle. Through the formula, it can be seen that $V_{n m}^{*}(\rho, \theta)$ can extract the features of images, and the high-frequency characteristics are obtained by $V_{n m}^{*}(\rho, \theta)$ for larger $n$ values. The lowfrequency characteristics are obtained by $V_{n m}^{*}(\rho, \theta)$ for smaller $n$ values. Zernike moments can be used to construct high moments. In theory, higher moments can contain more image information, so pattern recognition will be better.

3.2.2. Rotation of Zernike Moments. When the rotation angle of an image is $a$, the expression of its Zernike moments is as follows:

$$
\begin{aligned}
A_{n m}^{\prime} & =\frac{n+1}{\Pi} \iint \rho^{2} \leq 1 \\
& =A_{n m} \exp (-j m \alpha) .
\end{aligned}
$$

From the upper formula, it is shown that the Zernike moments' phases change, but the modulus remains unchanged. So, $\left|A_{n m}\right|$ can be regarded as the target rotation invariance feature.

3.3. Rough Set Neural Network. Rough set theory (RS theory) [22] was proposed by Pawlak, a mathematician of Poland in 1982. It is an effective method for dealing with uncertain, inconsistent, and imprecise problems. It can discover hidden knowledge from uncertainty and reveal its potential law. It defines knowledge from a new point of view, regards knowledge as a division of the universe, and holds that knowledge is granular. In recent years, the research on rough set theory in the field of artificial intelligence has been a hot topic, and this theory has been widely used. Knowledge reduction [23-25] is one of the core concepts of rough set theory.

3.3.1. Rough Set Principle. In rough set theory, knowledge is expressed in the form of an information table $S=(U, R, V, f)$, where $\mathrm{U}$ is a collection of objects, also called a domain, $R=C \cup D, \mathrm{CnD}=O, \mathrm{C}$ is called the conditional attribute set, $D$ is called the decision attribute set, $V \underset{r \in R}{=} U V_{r}$ is a collection of attribute values, $V_{r}$ represents the attribute value range of an attribute $r \in R$, and 
$f: U \times R \longrightarrow V$ is an information function that specifies the attribute value of each object $x$ in $\mathrm{U}$.

The simplification of a decision table is meant to simplify the conditional attributes in the decision table. The simplified decision table also has the function of simplifying the decision table before, and the simplified decision table has less conditional attributes. The rough set is used to describe the knowledge, and the reduction of redundant attributes can be used to simplify the knowledge. Finally, a complex decision table is reduced to the minimum conditional attribute decision table with no redundant attributes and the correct classification is ensured.

\subsubsection{Rough Set Neural Network (RS-BP Hybrid Model).} The RS-BP hybrid model is a hybrid model combining artificial neural network and rough set theory. The training time is too long when the neural network and the sample are both large. This inherent disadvantage is one of the main factors which restricts the further practicability of the neural network. Although various algorithms for improving training speed have emerged, the problem is far from being completely solved. Simplifying the training sample set and eliminating redundant data are another way to improve training speed. The rough set is used to simplify the neural network in order to train the sample set, and the redundant data is eliminated under the premise of retaining important information. Simulation results show that the training speed is 4.77 times better, and good results are achieved [26].

The characteristic parameters of the vibration are extracted through the condition monitoring system and a field test in the fault diagnosis process of the hydroelectric generating set. In order to describe the vibration mode of the hydrogenerator set as much as possible, more units are set up to extract the characteristic quantities of these units, some of which are related and some of which are independent. These feature parameters are often incomplete and redundant, which makes the modeling of subsequent fault diagnosis complex. The rough set theory can be used to reduce the knowledge of the training samples of neural network and get fewer attribute values while ensuring the goal of not missing important information.

\section{Simulation and Pattern Recognition of the Shaft Orbit}

Using Matlab to simulate the shaft orbits of the generator set when the fault occurs, formula (22) can be obtained:

$$
\begin{aligned}
& x(t)=A_{1} \sin \left(\omega t+\alpha_{1}\right)+A_{2} \sin \left(2 \omega t+\alpha_{2}\right), \\
& y(t)=B_{1} \cos \left(\omega t+\beta_{1}\right)+B_{2} \cos \left(2 \omega t+\beta_{2}\right),
\end{aligned}
$$

where $\omega$ is the angular frequency; $A_{1}, A_{2}, \alpha_{1}, \alpha_{2}$, $A_{1}, A_{2}, \alpha_{1}, \alpha_{2}$ are the amplitudes and initial phases of the first and second frequency components of $x(t)$ and $y(t)$. By changing the 8 parameters $A_{1}, A_{2}, \alpha_{1}, \alpha_{2}, B_{1}, B_{2}, \beta_{1}, \beta_{2}$, the shaft orbits of the hydroelectric unit are obtained, as shown in Figure 3. The 30 simulated shaft orbits on the left are training samples, and the 10 orbits on the right are test samples.

In this paper, $\mathrm{Hu}$ moments and Zernike moments are used to extract the features of shaft orbits. 7 features of the $\mathrm{Hu}$ moments are extracted and there are 6 improved $\mathrm{Hu}$ moments. Zernike moments can be used to construct high moments, and higher moments contain more image information.

In this paper, 49 features of Zernike moments can be obtained by choosing $0 \sim 12$ order Zernike moments. The $\mathrm{Hu}$ moments, improved $\mathrm{Hu}$ moments, and the data of the Zernike moments, after the rough set knowledge reduction is extracted from Figure 3, are shown in Tables 2-4. Tables 3 and 4 show the average values of the $\mathrm{Hu}$ moments and the improved $\mathrm{Hu}$ moments of typical shaft orbits.

The Hu moments and the Zernike moments are chosen as input variables, and the type of shaft orbit is used as the output in order to establish a neural network. The shapes of the shaft orbits can be identified by training the samples. For 49 Zernike moments, the input vector node is 49 , and the network structure is relatively complex, which will cause the training time to become longer. The rough set feature selection algorithm is used to reduce the input and remove redundant information. Finally, 13 Zernike moments' features are selected: A5.3, A6.6, A6.4, A6.2, A8.8, A8.4, A8.2, A9.7, A10.8, A12.10, A12.8, A12.6, and A12.4.

In order to fully reflect the influence of different feature quantities on pattern recognition, different feature input parameters are used for the pattern recognition of shaft orbits. The first method uses $7 \mathrm{Hu}$ moments as input variables. The second method uses 6 improved Hu moments as input variables. The third method takes the 49 moments as input variables. The fourth method uses the 13 moments eigenvalues reserved by the rough set feature selection algorithm as input variables. The neural network models of the four methods all adopt a three-layer BP neural network structure. The number of neurons in each input layer is as follows: $6,7,49$, and 13 . The hidden layer uses 10 neurons, and the output layer is 5 neurons, namely, five shaft orbits. The training step $=20000$, the descent gradient $=0.1$, and the error target $=0.001$. The flow chart of the fourth method is shown in Figure 4. Input samples and test samples are shown in Figure 4. The data for the sample training are shown in Tables 1, 5, and 6 . The results of training and recognition for the four neural networks are shown in Table 7.

It can be seen from Table 7 that Zernike moments are better than $\mathrm{Hu}$ moments for pattern recognition, and the former have the advantages of a small calculation error and a high recognition rate. Calculating the effects of Model 3 and Model 4, both have the same recognition accuracies and convergence rates, but the latter significantly reduces the calculation step. We can see that using the rough set method to advance the information reduction pretreatment is effective, and it can increase the convergence speed of the effect. The combination of Zernike moments, the rough set, and three neural networks can efficiently and accurately identify the shaft orbits, while the neural network with the Zernike moments and the rough set preprocessing can achieve good 


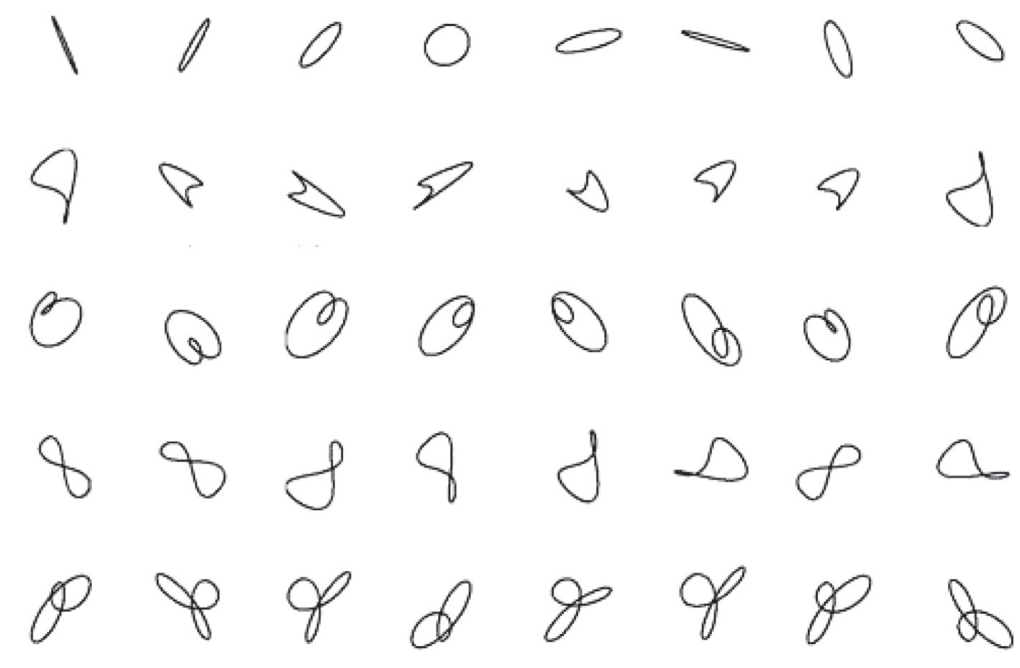

(a)

(b)

FIgURE 3: Simulation of axis orbits (ellipse, banana, inner 8, foreign 8, petal). (a) Training sample. (b) Testing sample.

TABLE 2: Hu moments of the simulated axis orbit.

\begin{tabular}{|c|c|c|c|c|c|c|c|}
\hline No. & Hul & $\mathrm{Hu} 2$ & Hu3 & $\mathrm{Hu} 4$ & Hu5 & Hu6 & Hu7 \\
\hline 1 & 0.187 & 0.363 & 4.309 & 4.602 & 9.067 & 4.510 & 9.735 \\
\hline 2 & 0.250 & 0.225 & 2.964 & 3.086 & 6.111 & 2.989 & 8.307 \\
\hline 3 & 0.116 & 0.177 & 3.931 & 5.038 & 9.610 & 5.157 & 9.764 \\
\hline 4 & 0.180 & 0.104 & 2.893 & 4.101 & 7.832 & 4.104 & 7.689 \\
\hline 5 & 0.270 & 1.442 & 3.162 & 2.984 & 6.393 & 3.928 & 6.108 \\
\hline 6 & 0.226 & 0.074 & 2.171 & 2.195 & 4.423 & 2.236 & 4.741 \\
\hline 7 & 0.231 & 0.271 & 3.461 & 3.280 & 6.864 & 3.189 & 6.751 \\
\hline 8 & 0.225 & 0.430 & 2.796 & 2.856 & 5.683 & 2.647 & 7.089 \\
\hline 9 & 0.279 & 0.190 & 0.268 & 2.957 & 4.412 & 3.160 & 4.500 \\
\hline 10 & 0.056 & 1.391 & 0.355 & 2.113 & 3.487 & 2.836 & 3.509 \\
\hline 11 & 0.078 & 0.514 & 0.386 & 1.501 & 2.451 & 1.836 & 3.219 \\
\hline 12 & 0.139 & 0.070 & 0.292 & 0.951 & 1.574 & 1.023 & 2.577 \\
\hline 13 & 0.156 & 0.103 & 0.430 & 1.100 & 1.894 & 1.219 & 2.318 \\
\hline 14 & 0.279 & 0.190 & 0.268 & 2.957 & 4.412 & 3.160 & 4.500 \\
\hline 15 & 0.056 & 1.391 & 0.355 & 2.113 & 3.487 & 2.836 & 3.509 \\
\hline 16 & 0.056 & 1.391 & 0.355 & 2.113 & 3.487 & 2.836 & 3.509 \\
\hline 17 & 0.162 & 1.603 & 1.099 & 0.874 & 1.945 & 2.749 & 2.104 \\
\hline 18 & 0.116 & 1.192 & 1.266 & 1.085 & 3.524 & 1.936 & 2.262 \\
\hline 19 & 0.150 & 0.847 & 1.234 & 1.007 & 2.157 & 1.519 & 2.571 \\
\hline 20 & 0.211 & 0.462 & 0.933 & 0.440 & 1.171 & 0.673 & 1.488 \\
\hline 21 & 0.166 & 0.264 & 1.132 & 0.568 & 1.425 & 0.712 & 2.159 \\
\hline 22 & 0.161 & 0.064 & 0.706 & 0.568 & 1.210 & 0.600 & 2.048 \\
\hline 23 & 0.166 & 0.264 & 1.132 & 0.568 & 1.425 & 0.712 & 2.159 \\
\hline 24 & 0.161 & 0.064 & 0.706 & 0.568 & 1.210 & 0.600 & 2.048 \\
\hline 25 & 0.251 & 0.303 & 2.300 & 3.880 & 8.691 & 3.744 & 6.970 \\
\hline 26 & 0.258 & 0.259 & 0.810 & 2.495 & 4.662 & 2.366 & 4.169 \\
\hline 27 & 0.267 & 0.230 & 0.384 & 2.012 & 4.185 & 1.909 & 3.212 \\
\hline 28 & 0.288 & 0.051 & 0.171 & 1.403 & 2.152 & 1.591 & 2.188 \\
\hline 29 & 0.246 & 0.079 & 0.023 & 2.965 & 4.507 & 3.093 & 4.809 \\
\hline 30 & 0.253 & 0.029 & 0.065 & 3.139 & 4.799 & 3.234 & 4.856 \\
\hline 31 & 0.262 & 0.031 & 0.154 & 2.905 & 5.400 & 3.155 & 4.282 \\
\hline 32 & 0.268 & 0.113 & 0.214 & 3.015 & 4.416 & 3.087 & 5.631 \\
\hline 33 & 0.131 & 0.055 & 0.510 & 1.125 & 2.138 & 1.485 & 2.057 \\
\hline 34 & 0.130 & 0.112 & 0.198 & 1.312 & 2.205 & 1.401 & 2.232 \\
\hline 35 & 0.095 & 0.409 & 0.033 & 1.317 & 2.043 & 1.925 & 2.205 \\
\hline 36 & 0.099 & 0.624 & 0.118 & 2.017 & 3.110 & 3.074 & 3.124 \\
\hline 37 & 0.131 & 0.055 & 0.510 & 1.125 & 2.138 & 1.485 & 2.057 \\
\hline 38 & 0.130 & 0.112 & 0.198 & 1.312 & 2.205 & 1.401 & 2.232 \\
\hline 39 & 0.095 & 0.409 & 0.033 & 1.317 & 2.043 & 1.925 & 2.205 \\
\hline 40 & 0.108 & 0.703 & 0.153 & 2.297 & 3.392 & 2.968 & 3.863 \\
\hline
\end{tabular}


TABLE 3: M moments of the simulated axis orbit.

\begin{tabular}{|c|c|c|c|c|c|c|}
\hline No. & M1 & M3 & M4 & M5 & M6 & M7 \\
\hline 1 & 0.01 & 9.71 & 10.29 & 10.16 & 5.24 & 10.82 \\
\hline 2 & 0.27 & 6.60 & 6.85 & 6.79 & 3.44 & 8.98 \\
\hline 3 & 0.06 & 8.39 & 10.61 & 10.14 & 5.51 & 10.30 \\
\hline 4 & 0.26 & 6.10 & 8.51 & 8.14 & 4.31 & 8.00 \\
\hline 5 & 1.98 & 2.00 & 1.64 & 2.07 & 1.04 & 1.78 \\
\hline 6 & 0.38 & 4.56 & 4.61 & 4.64 & 2.38 & 4.96 \\
\hline 7 & 0.19 & 7.74 & 7.37 & 7.68 & 3.73 & 7.56 \\
\hline 8 & 0.02 & 6.88 & 7.00 & 6.97 & 3.51 & 8.38 \\
\hline 9 & 0.75 & 1.11 & 5.34 & 3.84 & 2.78 & 3.93 \\
\hline 10 & 1.50 & 3.46 & 0.05 & 0.69 & 0.05 & 0.67 \\
\hline 11 & 0.67 & 0.77 & 1.46 & 0.91 & 0.81 & 1.68 \\
\hline 12 & 0.35 & 0.37 & 1.69 & 1.37 & 0.88 & 2.37 \\
\hline 13 & 0.21 & 1.17 & 2.51 & 2.20 & 1.42 & 2.63 \\
\hline 14 & 0.75 & 1.11 & 5.34 & 3.84 & 2.78 & 3.93 \\
\hline 15 & 1.50 & 3.46 & 0.05 & 0.69 & 0.05 & 0.67 \\
\hline 16 & 1.50 & 3.46 & 0.05 & 0.69 & 0.05 & 0.67 \\
\hline 17 & 1.93 & 2.61 & 3.06 & 2.86 & 0.46 & 2.70 \\
\hline 18 & 1.42 & 1.05 & 1.41 & 0.05 & 0.45 & 1.32 \\
\hline 19 & 1.15 & 0.07 & 0.53 & 0.38 & 0.17 & 0.03 \\
\hline 20 & 0.88 & 0.48 & 0.51 & 0.21 & 0.25 & 0.10 \\
\hline 21 & 0.60 & 1.47 & 0.34 & 0.63 & 0.18 & 1.37 \\
\hline 22 & 0.39 & 1.22 & 0.94 & 1.02 & 0.47 & 1.86 \\
\hline 23 & 0.60 & 1.47 & 0.34 & 0.63 & 0.18 & 1.37 \\
\hline 24 & 0.39 & 1.22 & 0.94 & 1.02 & 0.47 & 1.86 \\
\hline 25 & 0.20 & 5.51 & 8.67 & 9.60 & 4.35 & 7.88 \\
\hline 26 & 0.26 & 2.40 & 5.77 & 5.44 & 2.88 & 4.95 \\
\hline 27 & 0.30 & 1.46 & 4.71 & 4.88 & 2.37 & 3.90 \\
\hline 28 & 0.53 & 0.19 & 2.96 & 2.31 & 1.69 & 2.34 \\
\hline 29 & 0.41 & 0.28 & 6.17 & 4.74 & 3.25 & 5.04 \\
\hline 30 & 0.48 & 0.04 & 6.36 & 4.88 & 3.29 & 4.94 \\
\hline 31 & 0.56 & 0.40 & 5.72 & 5.31 & 3.09 & 4.19 \\
\hline 32 & 0.65 & 0.77 & 5.69 & 4.08 & 2.86 & 5.29 \\
\hline 33 & 0.32 & 0.85 & 2.09 & 1.97 & 1.37 & 1.89 \\
\hline 34 & 0.37 & 0.06 & 2.29 & 1.87 & 1.18 & 1.90 \\
\hline 35 & 0.60 & 1.29 & 1.41 & 0.82 & 1.11 & 0.98 \\
\hline 36 & 0.82 & 2.11 & 2.16 & 1.24 & 1.83 & 1.25 \\
\hline 37 & 0.32 & 0.85 & 2.09 & 1.97 & 1.37 & 1.89 \\
\hline 38 & 0.37 & 0.06 & 2.29 & 1.87 & 1.18 & 1.90 \\
\hline 39 & 0.60 & 1.29 & 1.41 & 0.82 & 1.11 & 0.98 \\
\hline 40 & 0.92 & 2.41 & 2.49 & 1.28 & 1.56 & 1.75 \\
\hline
\end{tabular}

results in the identification of shaft orbits. Zernike moments can extract the features of shaft orbits, and the rough set method is used to solve the redundancy problem, while the neural network solves the problem of sensitivity to noise. Therefore, by integrating the advantages of Zernike moments, rough sets, and neural networks, the efficiency and accuracy of pattern recognition can be greatly improved. 
TABLE 4: Zernike moments of the simulated axis orbit.

\begin{tabular}{|c|c|c|c|c|c|c|c|c|c|c|c|c|c|}
\hline No. & A5.3 & A6.6 & A6.4 & A6.2 & A8.8 & A8.4 & A8.2 & A9.7 & A10.8 & A12.10 & A12.8 & A12.6 & A12.4 \\
\hline 1 & 55.0 & 6.0 & 39.6 & 35.3 & 1.2 & 46.9 & 33.6 & 2.0 & 36.6 & 59.1 & 26.2 & 17.8 & 5.6 \\
\hline 2 & 24.6 & 49.3 & 22.4 & 14.2 & 9.2 & 5.0 & 5.6 & 5.6 & 12.0 & 16.2 & 23.0 & 3.8 & 15.2 \\
\hline 3 & 14.2 & 5.2 & 5.2 & 25.8 & 1.4 & 8.2 & 28.3 & 2.9 & 31.0 & 6.0 & 8.0 & 27.6 & 11.3 \\
\hline 4 & 12.6 & 11.8 & 3.2 & 25.6 & 0.8 & 10.4 & 23.5 & 1.3 & 7.7 & 1.3 & 14.3 & 22.0 & 2.1 \\
\hline 5 & 18.3 & 19.8 & 1.5 & 15.1 & 1.3 & 16.1 & 14.0 & 3.6 & 10.0 & 5.2 & 21.6 & 13.4 & 10.3 \\
\hline 6 & 17.5 & 28.9 & 16.2 & 5.3 & 0.6 & 7.8 & 9.1 & 0.7 & 3.9 & 17.7 & 14.8 & 3.3 & 14.7 \\
\hline 7 & 32.5 & 20.4 & 2.2 & 4.4 & 1.3 & 1.6 & 14.9 & 0.5 & 8.9 & 9.8 & 9.8 & 12.6 & 23.4 \\
\hline 8 & 30.1 & 4.9 & 5.0 & 21.8 & 1.0 & 3.7 & 28.9 & 0.5 & 11.0 & 2.9 & 8.7 & 21.8 & 5.5 \\
\hline 9 & 19.2 & 10.1 & 6.6 & 10.7 & 5.0 & 9.8 & 5.8 & 9.4 & 4.5 & 12.4 & 6.2 & 7.2 & 11.2 \\
\hline 10 & 27.1 & 9.7 & 3.8 & 29.5 & 13.9 & 1.3 & 28.0 & 4.8 & 11.7 & 14.0 & 5.1 & 17.5 & 15.1 \\
\hline 11 & 24.3 & 33.3 & 19.2 & 8.8 & 6.6 & 14.7 & 9.3 & 7.4 & 3.2 & 19.7 & 24.6 & 6.5 & 20.7 \\
\hline 12 & 14.4 & 25.3 & 26.1 & 7.7 & 6.6 & 13.1 & 5.7 & 5.4 & 12.1 & 29.3 & 6.0 & 10.0 & 15.6 \\
\hline 13 & 28.0 & 7.5 & 6.6 & 17.4 & 6.1 & 6.9 & 22.2 & 6.9 & 13.2 & 8.6 & 7.1 & 12.2 & 23.7 \\
\hline 14 & 32.0 & 7.4 & 13.5 & 17.7 & 40.2 & 41.8 & 4.0 & 7.6 & 4.1 & 7.4 & 20.3 & 11.6 & 12.4 \\
\hline 15 & 26.2 & 15.4 & 7.5 & 21.1 & 13.1 & 0.9 & 27.9 & 3.9 & 10.4 & 6.5 & 7.6 & 10.1 & 9.0 \\
\hline 16 & 27.1 & 9.7 & 3.8 & 29.5 & 13.9 & 1.3 & 28.0 & 4.8 & 11.7 & 14.0 & 5.1 & 17.5 & 15.1 \\
\hline 17 & 1.9 & 10.7 & 16.0 & 16.4 & 1.9 & 4.7 & 18.6 & 17.0 & 6.3 & 23.9 & 13.7 & 10.5 & 8.2 \\
\hline 18 & 15.2 & 6.8 & 25.8 & 18.6 & 13.8 & 20.3 & 26.4 & 26.3 & 2.6 & 20.6 & 19.7 & 25.8 & 6.2 \\
\hline 19 & 6.5 & 2.3 & 38.2 & 6.3 & 7.7 & 16.7 & 12.5 & 11.6 & 12.0 & 27.6 & 14.1 & 5.3 & 7.9 \\
\hline 20 & 7.8 & 14.7 & 14.1 & 5.3 & 9.1 & 10.3 & 2.2 & 0.9 & 0.6 & 8.6 & 1.4 & 7.4 & 12.0 \\
\hline 21 & 9.6 & 14.7 & 15.2 & 5.6 & 6.8 & 4.6 & 4.0 & 11.1 & 13.6 & 18.8 & 8.2 & 1.5 & 7.2 \\
\hline 22 & 17.1 & 18.0 & 16.6 & 3.9 & 7.1 & 9.6 & 4.3 & 8.9 & 1.9 & 16.6 & 4.6 & 2.3 & 0.7 \\
\hline 23 & 18.8 & 5.3 & 20.9 & 20.7 & 25.1 & 10.0 & 14.2 & 28.3 & 14.6 & 17.7 & 31.6 & 29.9 & 20.4 \\
\hline 24 & 16.4 & 7.2 & 25.0 & 5.8 & 2.4 & 20.2 & 14.5 & 16.1 & 5.9 & 4.5 & 4.7 & 27.7 & 26.3 \\
\hline 25 & 2.8 & 38.3 & 7.4 & 4.8 & 13.8 & 17.2 & 0.9 & 0.5 & 5.2 & 12.3 & 17.1 & 16.4 & 17.1 \\
\hline 26 & 12.0 & 35.4 & 5.7 & 13.8 & 4.5 & 4.3 & 18.4 & 2.4 & 11.1 & 22.3 & 22.2 & 1.0 & 13.2 \\
\hline 27 & 24.6 & 8.8 & 7.4 & 16.3 & 3.6 & 6.7 & 21.6 & 2.9 & 5.7 & 2.1 & 15.6 & 16.6 & 14.8 \\
\hline 28 & 18.0 & 26.2 & 7.4 & 7.6 & 13.6 & 18.3 & 8.1 & 0.6 & 8.3 & 7.6 & 9.0 & 3.9 & 1.7 \\
\hline 29 & 16.3 & 16.2 & 5.2 & 11.4 & 12.1 & 34.7 & 13.2 & 4.8 & 5.0 & 2.8 & 23.8 & 20.3 & 21.8 \\
\hline 30 & 26.1 & 14.7 & 4.5 & 4.2 & 3.4 & 5.7 & 13.4 & 1.7 & 6.8 & 5.4 & 14.2 & 14.5 & 17.8 \\
\hline 31 & 27.5 & 29.1 & 10.4 & 2.0 & 8.8 & 14.6 & 6.5 & 12.5 & 11.4 & 12.4 & 5.2 & 1.2 & 7.8 \\
\hline 32 & 19.4 & 13.9 & 9.0 & 10.8 & 11.8 & 6.6 & 8.0 & 11.1 & 12.9 & 23.8 & 6.9 & 5.2 & 13.4 \\
\hline 33 & 19.4 & 18.8 & 14.7 & 5.9 & 4.6 & 7.4 & 6.2 & 3.5 & 1.7 & 20.4 & 11.3 & 2.5 & 8.0 \\
\hline 34 & 11.0 & 23.8 & 20.8 & 1.6 & 4.8 & 3.1 & 10.1 & 9.4 & 12.3 & 16.1 & 5.2 & 10.7 & 1.9 \\
\hline 35 & 4.3 & 20.7 & 25.6 & 4.9 & 8.9 & 5.2 & 7.1 & 17.1 & 13.6 & 18.1 & 20.5 & 5.2 & 13.6 \\
\hline 36 & 10.6 & 9.8 & 27.3 & 3.9 & 1.4 & 8.2 & 11.6 & 20.1 & 11.9 & 17.7 & 8.2 & 4.5 & 0.9 \\
\hline 37 & 19.4 & 18.8 & 14.7 & 5.9 & 4.6 & 7.4 & 6.2 & 3.5 & 1.7 & 20.4 & 11.3 & 2.5 & 8.0 \\
\hline 38 & 5.9 & 11.5 & 19.2 & 4.2 & 13.4 & 12.7 & 1.2 & 5.2 & 8.2 & 7.4 & 26.0 & 12.9 & 1.6 \\
\hline 39 & 5.8 & 11.6 & 11.9 & 3.4 & 6.5 & 19.0 & 9.8 & 7.3 & 5.8 & 21.8 & 24.5 & 1.5 & 2.3 \\
\hline 40 & 6.7 & 13.2 & 25.1 & 10.9 & 0.5 & 7.0 & 9.9 & 17.0 & 7.3 & 23.6 & 8.0 & 4.4 & 4.5 \\
\hline
\end{tabular}




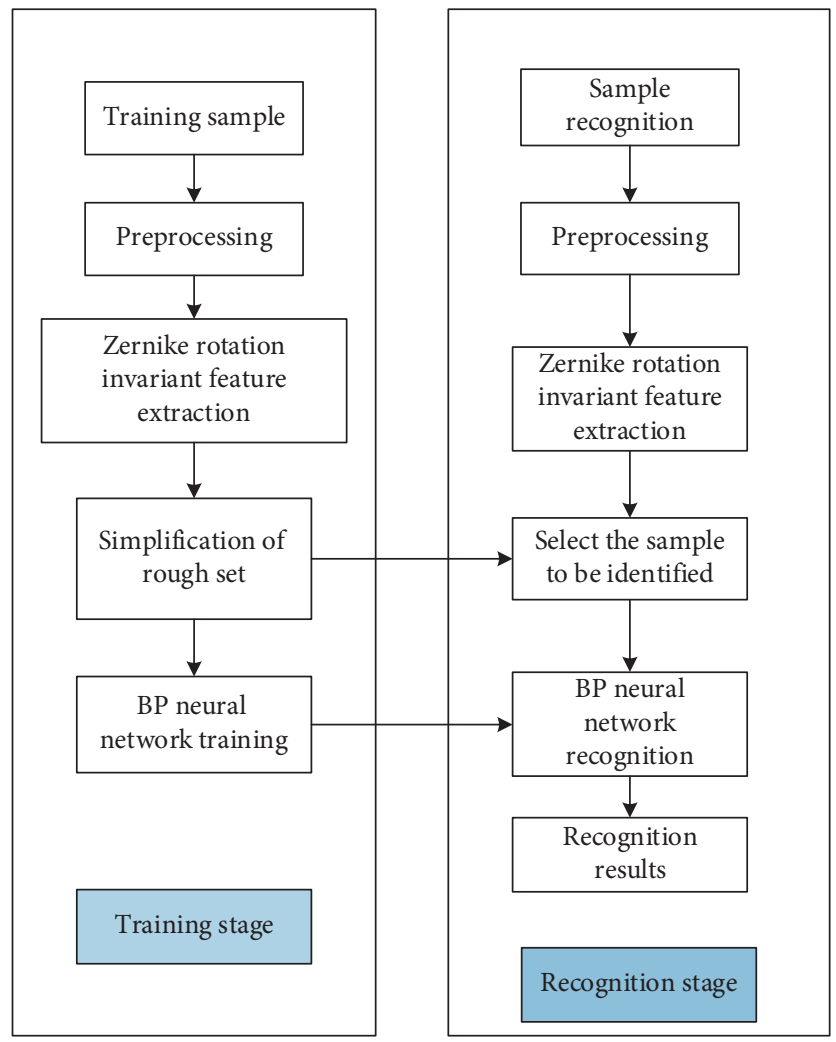

FIGURE 4: Flowchart of the rough set neural network model.

Table 5: Average values of $\mathrm{Hu}$ moments.

\begin{tabular}{lccccccc}
\hline Axis orbit & $\bar{\phi}_{1}$ & $\bar{\phi}_{2}$ & $\bar{\phi}_{3}$ & $\bar{\phi}_{4}$ & $\bar{\phi}_{5}$ & $\bar{\phi}_{6}$ \\
\hline Ellipse & 0.211 & 0.386 & 3.211 & 3.518 & 6.998 & 3.595 \\
Banana & 0.137 & 0.655 & 0.339 & 1.976 & 3.150 & 2.363 \\
Inner 8 & 0.162 & 0.595 & 1.026 & 0.710 & 1.758 & 1.188 \\
Outer "8" & 0.262 & 0.137 & 0.515 & 2.727 & 4.852 & 2.772 \\
Petal & 0.115 & 0.310 & 0.219 & 1.478 & 2.409 & 1.953 \\
\hline
\end{tabular}

TABLE 6: Average values of improved Hu moments.

\begin{tabular}{lcccccc}
\hline Axis orbit & $\bar{M}_{1}^{*}$ & $\bar{M}_{3}^{*}$ & $\bar{M}_{4}^{*}$ & $\bar{M}_{5}^{*}$ & $\bar{M}_{6}^{*}$ \\
\hline Ellipse & 0.396 & 6.497 & 7.111 & 7.073 & 3.645 \\
Banana & 0.904 & 1.864 & 2.063 & 1.778 & 1.105 \\
Inner 8 & 0.919 & 1.199 & 1.009 & 0.852 & 0.330 \\
Outer "8" & 0.422 & 1.382 & 5.756 & 5.154 & 2.066 \\
Petal & 0.540 & 1.117 & 2.026 & 1.480 & 1.325 \\
\hline
\end{tabular}

TABLE 7: Statistics of training and recognition results of different recognition methods.

\begin{tabular}{lcccc}
\hline Serial number & Input & Time step & Error & Recognition rate (\%) \\
\hline Model 1 & Hu moments (7 variables) & 20000 & 0.00104 & 80 \\
Model 2 & Improved Hu moments (6 variables) & 20000 & 0.00131 & 80 \\
Model 3 & Zernike moments (49 variables) & 13153 & 0.001 & 90 \\
Model 4 & Zernike moments (13 variables) & 9358 & 0.001 & 90 \\
\hline
\end{tabular}




\section{Conclusions}

In this paper, the feature extraction and pattern recognition of the shaft orbit of a hydroelectric generating unit are studied. The main content and conclusions are as follows:

(1) In terms of shaft orbits feature extraction and pattern recognition, the Zernike moment is better than the $\mathrm{Hu}$ moment and it has the advantages of a small calculation error and a high recognition rate.

(2) A rough set neural network (RS-BP hybrid model) of shaft orbit recognition is established, which uses just 13 moment eigenvalues reserved by the rough set feature selection algorithm as input variables, has the same calculation error and recognition rate, and reduces the calculation time step. The simulation of the recognition of shaft orbits shows that the hybrid model has achieved good results in the identification of shaft orbits.

\section{Data Availability}

No data were used to support this study.

\section{Conflicts of Interest}

The authors declare that they have no conflicts of interest.

\section{Acknowledgments}

This project was supported by the Central University Fundamental Research Funds (2015B02814), the IWHR Research and Development Support Program (HM0145B182017), and the Key Project of Yunnan Power Grid Co. Ltd. (YNYJ2016043).

\section{References}

[1] Z. Han, R. He, and Y. Xu, "Study on resonance mechanism of power system low frequency oscillation induced by turbopressure pulsation," Proceedings of the Chinese Society of Electrical Engineering, vol. 59, pp. 47-51, 2008.

[2] Z. Han, R. He, and Y. Xu, "Power system low frequency oscillation of resonance mechanism induced by turbopressure pulsation," Proceedings of the Chinese Society of Electrical Engineering, vol. 7, pp. 14-18, 2005.

[3] H. Zhang and Y. Bai, "A smart diagnosis system based on automatic recognition of multiple rotor faults," Advances in Mechanical Engineering, vol. 9, 2017.

[4] N. S. Vyas and D. Satishkumar, "Artificial neural network design for fault identification in a rotor-bearing system," Mechanism and Machine Theory, vol. 36, no. 2, pp. 157-175, 2001.

[5] S. Tang, S. Yuan, and Y. Zhu, "Deep learning-based intelligent fault diagnosis methods toward rotating machinery," IEEE Access, vol. 8, no. 1, pp. 9335-9346, 2020.

[6] X. Xiang, J. Zhou, X. An, B. Peng, and J. Yang, "Fault diagnosis based on Walsh transform and support vector machine," Mechanical Systems and Signal Processing, vol. 22, no. 7, pp. 1685-1693, 2008.
[7] H. Jeong, S. Park, S. Woo, and S. Lee, "Rotating machinery diagnostics using deep learning on orbit plot images," Procedia Manufacturing, vol. 5, pp. 1107-1118, 2016.

[8] B. Fu, J. Zhou, and W. Chen, Identification of the Shaft Orbits for Turbine Rotor by Modified Fourier Descriptors, Institute of Electrical and Electronics Engineers Inc., Shanghai, China, 2004.

[9] B. Fu, J. Zhou, and W. Chen, "Method based on Fourier descriptors to recognize shaft orbit," Dianli Xitong Zidonghua/Automation of Electric Power Systems, vol. 28, no. 12, pp. 40-44, 2004.

[10] D. F. Shi, W. J. Wang, P. J. Unsworth, and L. S. Qu, "Purification and feature extraction of shaft orbits for diagnosing large rotating machinery," Journal of Sound and Vibration, vol. 279, no. 3-5, pp. 581-600, 2005.

[11] J. J. Sinou and A. W. Lees, "The influence of cracks in rotating shafts," Journal of Sound and Vibration, vol. 285, no. 4-5, pp. 1015-1037, 2005.

[12] F. Xu, B. Zhong, and R. Huang, "Automatic recognition of shaft orbit and its application to fault diagnosis of rotating machinery," Zhendong Ceshi Yu Zhenduan/Journal of Vibration, Measurement and Diagnosis, vol. 29, no. 2, pp. 141145, 2009.

[13] B. Fu, J. Zhou, and B. Peng, "Auto-recognition of shaft orbits using affine moments invariants," Huazhong Keji Daxue Xuebao (Ziran Kexue Ban)/Journal of Huazhong University of Science and Technology (Natural Science Edition), vol. 35, no. 3, pp. 119-122, 2007.

[14] P. Guo, H. Li, and J. Yuan, "Intelligence identification for multi-class shaft centerline orbit of hydropower unit based on improved SVM model," Nongye Gongcheng Xuebao/Transactions of the Chinese Society of Agricultural Engineering, vol. 29, no. 15, pp. 65-71, 2013.

[15] X. Hu, G. Luo, and D. Gao, "Numerical analysis and experiment of counter-rotating dual-rotor's steady-state response," Hangkong Dongli Xuebao/Journal of Aerospace Power.vol. 22, no. 7, pp. 1044-1049, 2007.

[16] Y. Yang, L. Zhou, J. Hang, D. Du, W. Shi, and Z. He, "Energy characteristics and optimal design of diffuser meridian in an electrical submersible pump," Renewable Energy, vol. 167, pp. 718-727, 2021.

[17] B. Fu, J. Zhou, and B. Peng, "Auto-recognition of shaft orbits rasing affine moments invariants," Journal of Huazhong University of Science and Technology Nature Science, vol. 35, no. 1671-4512, pp. 119-122, 2007.

[18] H. Kauppinen, T. Seppanen, and M. Pietikainen, "An experimental comparison of autoregressive and Fourier-based descriptors in 2D shape classification," IEEE Transactions on Pattern Analysis and Machine Intelligence, vol. 17, no. 2, pp. 201-207, 1995.

[19] M. Hu, "Visual pattern recognition by moments invariants," Information Theory Ire Transactions on, vol. 8, no. 2, pp. 179-187, 1962.

[20] R. Y. Wong and E. L. Hall, "Scene matching with invariant moments," Computer Graphics and Image Processing, vol. 8, no. 1, pp. 16-24, 1978.

[21] v. F. Zernike, "Beugungstheorie des schneidenver-fahrens und seiner verbesserten form, der phasenkontrastmethode," Physica, vol. 1, no. 7-12, pp. 689-704, 1934.

[22] Z. Pawlak, "Rough sets," International Journal of Computer and Information Sciences, vol. 11, pp. 11-18, 1982.

[23] C. Yang, H. Liu, S. Mcloone, C. L. P. Chen, and X. Wu, “A novel variable precision reduction approach to 
comprehensive knowledge systems," IEEE Transactions on Cybernetics, vol. 48, no. 2, pp. 661-674, 2018.

[24] Y. Jing, T. Li, H. Fujita, Z. Yu, and B. Wang, "An incremental attribute reduction approach based on knowledge granularity with a multi-granulation view," Information Sciences, vol. 411, pp. 23-38, 2017.

[25] G. Wu, H. Wu, and T. Huang, "New method of knowledge reduction based on fusion of evidence theory and entropy," Computer Engineering and Application, vol. 52, no. 1002-8331, pp. 167-170, 2016.

[26] L. Bai, L. Zhou, X. Jiang, Q. Pang, and D. Ye, "Vibration in a multistage centrifugal pump under varied conditions," Shock and Vibration, vol. 2019, pp. 1-9, Article ID 2057031, 2019. 